arcs of classes $X, Y$ or $Z$. The order relation on a Jordan curve is the same as that on the circumference of a circle. Hence, when a Jordan curve of $J(r)$ is deformed continuously on $F$, the number of arcs of $\mathscr{L}_{1}$ resulting which are of class $Z$ (that is, the number of arcs lying in $S_{2}$ which connect $S_{3}, S_{4}$ ) can only change by an even number. But curves of type (i) can be deformed to a point and so must give rise to an even number of arcs of class $Z$ in $\mathcal{L}_{1}$. Also curves of type (ii) can be deformed to $J_{0}$ which has a single arc in $S_{2}$ joining $S_{3}, S_{4}$. Hence these must give rise to an odd number of arcs of class $Z$ in $\mathscr{L}_{1}$.

However we have seen that there is an odd number of curves of type (ii) in $J(r)$. Hence there can only be an odd number of arcs of class $Z$ in $\mathcal{L}_{1}$. By symmetry the same result is true for $\mathcal{L}_{2}, \mathcal{L}_{3}, \mathscr{L}_{4}$; so that condition (ii) in the definition of a pseudo-square $(\S 2)$ is satisfied by $Q(r)$.

Further

$$
\begin{aligned}
Q-\rho\left(\mathcal{L}_{1}, \mathcal{L}_{3}\right) & \geqslant \mathrm{e}_{-\rho}\left(\mathcal{L}_{1}, \mathcal{L}_{3}\right) \text { by }(1) \\
& \geqslant e_{-\rho}\left(S_{2}, S_{5}\right)=b \text { by }(2),
\end{aligned}
$$

and

$$
Q-\rho\left(\mathscr{L}_{2}, \mathscr{L}_{4}\right) \geqslant c \text {. }
$$

Thus $Q(r)$ is a pseudo-square of dimensions at least $b, c$; and therefore, by Lemma 2 , the area of $Q(r)$ is at least $b c$.

A similar argument to that of Lemma 1 will now prove that

$$
\Lambda^{3} G(a) \geqslant b c(a-\epsilon),
$$

and the theorem is proved, since $\epsilon$ is arbitrary and $\odot \supset G(a)$.

I am grateful to Prof. Besicovitch for giving me the manuscript of his paper before publication, and for pointing out to me some obscurities in my proof.

Peterhouse, Cambridge.

\title{
ON MAHLER'S $U$-NUMBERS
}

\section{W. J. LEVEQUE†.}

1. It is well known that a necessary and sufficient condition that the complex number $\xi$ be transcendental is that to each $\omega>0$ there correspond a positive integer $n=n(\omega)$ and infinitely many sets of rational integers $a_{0}, \ldots, a_{n}$, such that

$$
0<\left|a_{0}+a_{1} \xi+\ldots+a_{n} \xi^{n}\right|<A^{-\omega},
$$

where $A=\max _{i}\left(\left|a_{i}\right|\right)$. In general, $n$ increases with $\omega$; the numbers $\xi$ for which $n$ may remain bounded form an uncountable set of measure zero which K. Mahler [1] has termed the $U$-numbers. The Liouville numbers 
are precisely the $U$-numbers for which $n$ can always be chosen to be unity; while they have been studied more or less extensively, no general investigation has heretofore been made of the $U$-numbers of higher "degree". (See, however, [2], pp. 143-148.)

We shall say that the polynomials

$$
f_{n}(x)=a_{0}^{(n)}+\ldots+a_{m}^{(n)} x^{m} \quad(n=1,2, \ldots)
$$

of fixed degree $m$ and height $H\left(f_{n}\right)=\max \left(\left|a_{0}^{(n)}\right|, \ldots,\left|a_{m}^{(n)}\right|\right)$ form a sequence of Liouville polynomials (more briefly, an $L_{m}$-sequence) for $\xi$ if

$$
H\left(f_{1}\right) \leqslant H\left(f_{2}\right) \leqslant \ldots
$$

and

$$
0<\left|f_{n}(\xi)\right|=H^{-\omega_{n}}\left(f_{n}\right)<1 \quad(n=1,2, \ldots),
$$

where $\lim _{n \rightarrow \infty} \omega_{n}=\infty$. We designate by $U$ the set of all $U$-numbers, and by $U_{m}$ the set of all $\xi$ for which there exists an $L_{m}$-sequence but not an $L_{m-1}$-sequence. Thus $U_{1}$ is the set of Liouville numbers, and $U$ is the union of all the (disjoint) sets $U_{m}$. An element of $U_{m}$ does not, of course, have a unique $L_{m}$-sequence, since any infinite subsequence of an $L_{m}$-sequence is again an $L_{m}$-sequence, but it is shown in Theorem 4 that there is at most one sequence satisfying certain auxiliary conditions. This result is used to give a constructive proof that $U_{m}$ is not empty for any $m \geqslant 1$, and an extension of it is used to show that numbers satisfying a transcendency condition due to $T$. Schneider are not $U$-numbers of too low degree.

2. Theorem 1. Suppose that the sequence $\left\{f_{n}(x)\right\}$ of polynomials (1) is an $L_{m}$-sequence for each of the distinct numbers $\xi_{1}, \ldots, \xi_{m}$. Then there are numbers $\alpha_{0}, \ldots, \alpha_{m}$ such that

$$
\lim _{n \rightarrow \infty} \frac{a_{k}^{(n)}}{a_{m}^{(n)}}=\alpha_{k} \quad(k=0, \ldots, m-1) ;
$$

they are either Liouville numbers or rational, and one at least is Liouville. If $\alpha_{k}$ is rational, then $a_{k}^{(n)} / a_{m}^{(n)}=\alpha_{k}$ for large $n$, while if $\alpha_{k} \varepsilon U_{1}$, then $a_{k}^{(n)} / a_{m}^{(n)}$ (or $p_{k}^{(n)} / q_{k}^{(n)}$, in reduced form) is a convergent to $\alpha_{k}$ for large $n$. Finally the monotonic union sequence $\left\{q^{(n)}\right\}$ (consisting of all the integers $t$ such that $t=q_{k}^{(n)}$ for some $k, n$, arranged by size) is a large-gap sequence; i.e.

$$
\limsup _{n \rightarrow \infty} \frac{\log q^{(n+1)}}{\log q^{(n)}}=\infty .
$$

For putting $\quad f_{n}(x)=a_{m}^{(n)}\left(x-\xi_{1}^{(n)}\right) \ldots\left(x-\xi_{m}^{(n)}\right)$

and using the fact that $f_{n}\left(\xi_{i}\right) \rightarrow 0$, we have $\xi_{i}^{(n)} \rightarrow \xi_{i}$ for $1 \leqslant i \leqslant m$ (after proper ordering), and so there exists

$$
\lim _{n \rightarrow \infty} \frac{f_{n}(x)}{a_{m}^{(n)}}=\lim _{n \rightarrow \infty}\left(\frac{a_{0}^{(n)}}{a_{m}^{(n)}}+\ldots+\frac{a_{m-1}^{(n)}}{a_{m}^{(n)}} x^{m-1}+x^{m}\right)=\alpha_{0}+\ldots+\alpha_{m-1} x^{m-1}+x^{m}
$$


We reserve the notation $\left\{f_{n}^{*}\right\}$ for the use to which it is put in this theorem, that is, to designate an $L$-sequence for $\xi$ derived from a defining equation for $\xi$ by replacing the coefficients by various of their convergents.

In the proofs of the next two theorems we shall need the following lemma :

Lemma. Suppose that $\phi(x), \phi_{1}(x)$ are polynomials with integral coefficients, of degrees $m, m_{1}$ respectively, that $\phi\left(\xi_{1}\right)=\ldots=\phi\left(\xi_{m}\right)=0$ and that $\phi_{1} \mid \phi$. Then

$$
\max _{k}\left(\left|\xi_{k}\right|\right) \leqslant 2 H(\phi)
$$

and there is a $c_{7}$, depending only on $m_{1}$, such that $\dagger$

$$
H\left(\phi_{1}\right)<c_{7} H^{m_{1}+1}(\phi) \text {. }
$$

We have, if $\phi(x)=a x^{m}+\ldots$ and $|x| \geqslant 2$,

$$
\left|\frac{\phi(x)}{a}\right| \geqslant|x|^{m}-H(\phi)\left(1+|x|+\ldots+|x|^{m-1}\right) \geqslant|x|^{m}-2 H(\phi)|x|^{m-1},
$$

so that either $\left|\xi_{k}\right|<2 \leqslant 2 H(\phi)$ or $0 \geqslant\left|\xi_{k}\right|-2 H(\phi)$, and (6) holds. Since the leading coefficient of $\phi_{1}$ divides that of $\phi$, and since the coefficients of $\phi_{1}$ are symmetric functions in certain of the zeros of $\phi$, we have by (6),

$$
\begin{aligned}
H\left(\phi_{1}\right) & \leqslant H(\phi) \cdot \max \left(\Sigma\left|\xi_{1}\right|, \ldots, \Sigma\left|\xi_{1} \ldots \xi_{m_{1}}\right|\right) \\
& <c_{7} 2^{-m_{1}} H(\phi) \cdot 2^{m_{1}} H^{m_{1}}(\phi),
\end{aligned}
$$

and the proof is complete.

Theorem 3. A necessary condition that $\xi$ belong to $U_{m}$ is that only finitely many elements of any $L_{m}$-sequence for $\xi$ be reducible.

Let $\left\{f_{n}\right\}$ be an $L_{m^{-}}$-sequence, with $\left|f_{n}(\xi)\right|=H^{-\omega_{n}}\left(f_{n}\right)$. Suppose that there is an infinite sequence $\left\{\phi_{n_{k}}\right\}$ of polynomials with integral coefficients such that $\phi_{n_{k}} \mid f_{n_{k}}, \operatorname{deg} \phi_{n_{k}}=m_{1}<m$, and $\left|\phi_{n_{k}}(\xi)\right| \rightarrow 0$. Then by the lemma, $H\left(\phi_{n_{k}}\right)<c_{7} H^{m_{1}+1}\left(f_{n_{k}}\right)$, and so

$$
\left|\phi_{n_{k}}(\xi)\right|<c_{8}\left|f_{n_{k}}(\xi)\right|^{1 / m}<c_{8}\left(H\left(\phi_{n_{k}}\right) / c_{7}\right)^{-\omega_{n} / m\left(m_{1}+1\right)},
$$

so that $\left\{\phi_{n_{k}}\right\}$ is an $L_{m_{1}}$-sequenc for $\xi$.

THEOREM 4. Suppose the $\xi$ is a complex number to which corresponds a sequence of polynomials $F_{n}(x)$ with the following properties:

1. $F_{n}(x)=a_{0}^{(n)}+\ldots+a_{m_{n}}^{n)} x^{m_{n}}=a_{m_{n}}^{(n)}\left(x-\xi^{(n)}\right)\left(x-\xi_{2}^{(n)}\right) \ldots\left(x-\xi_{m_{n}}^{(n)}\right)$,

where the numbers $a_{k}^{(n)}$ are integers, the zeros of $F_{n}$ have been so ordered that $\xi_{1}^{(n)}=\xi^{(n)}$ is the zero nearest to $\xi$, and $H\left(F_{1}\right) \leqslant H\left(F_{2}\right) \leqslant \ldots$.

† C. L. Siegel, Math. Zeitschrift, 10 (1921), 716, has given the incomparably better bound $H\left(\phi_{1}\right)<\frac{m !}{m_{1} l} B(\phi)$, but the trivial result of the lemma suffices here. 
2. $\left|F_{n}(\xi)\right|=H^{-\omega_{n}}\left(F_{n}\right)$, where

$$
\lim _{n \rightarrow \infty} \omega_{n}=\infty
$$

3. All the $F_{n}$ are irreducible.

4. For some $m, m_{n} \leqslant m$ for all $n$.

5. For some $\delta>0, \quad H^{\omega_{n}}\left(F_{n}\right)>H^{\delta}\left(F_{n+1}\right)$.

Then this sequence is unique, in the sense that if $\left\{f_{v}\right\}$ is any $L_{r}$-sequence ( $r$ an arbitrary positive integer) for $\xi$, then for sufficiently large values of $\nu$, $f_{\nu}$ is divisible by some $F_{n}$.

Clearly

$$
\left|\xi-\xi^{(n)}\right|<c_{9} H^{-\omega_{n} / m}\left(F_{n}\right)
$$

so that we can put

$$
\xi=\xi^{(n)}+t_{n} H^{-\omega_{n} / m}\left(F_{n}\right),
$$

where $\left|t_{n}\right|<c_{9}$ for all $n$.

Now put

$$
f_{v}(x)=d_{r}^{(\nu)} x^{r}+\ldots+d_{0}^{(\nu)}=d_{r}^{(\nu)}\left(x-\vartheta_{1}^{(\nu)}\right) \ldots\left(x-\vartheta_{r}^{(\nu)}\right) .
$$

Then from the relation

$$
\left(a_{m_{n}}^{(n)}\right)^{r}\left(d_{r}^{(\nu)}\right)^{m_{n}} \prod_{\substack{1 \leqslant i \leqslant r \\ 1 \leqslant j \leqslant m_{n}}}\left(\vartheta_{i}^{(\nu)}-\xi_{j}^{(n)}\right)=\left(a_{m_{n}}^{(n)}\right)^{r} \prod_{i=1}^{r} F_{n}\left(\vartheta_{i}^{(\nu)}\right)=\left(d_{r}^{(\nu)}\right)^{m_{n}} \prod_{i=1}^{m_{n}} f_{\nu}\left(\xi_{i}^{(n)}\right),
$$

we obtain the identity

$$
\left|f_{v}\left(\xi^{(n)}\right)\right|=\frac{\left|\left(d_{r}^{(v)}\right)^{m_{n}} \prod_{i=1}^{r} F_{n}\left(\vartheta_{i}^{(\nu)}\right)\right|}{\left|\left(a_{m_{n}}^{(n)}\right)_{i=2}^{r} \prod_{i=2}^{m_{n}} \frac{f_{v}\left(\xi_{i}^{(n)}\right)}{H\left(f_{v}\right)}\right| \cdot H^{m_{n}-1}\left(f_{v}\right)}
$$

The numerator on the right is an integer, and we have the bounds

$$
\begin{gathered}
\left|a_{m_{n}}^{\left(n_{n}\right)}\right|^{r} \leqslant H^{r}\left(F_{n}\right), \\
\prod_{i=2}^{m_{n}}\left|\frac{f_{v}\left(\xi_{i}^{(n)}\right)}{H\left(f_{\nu}\right)}\right| \leqslant \prod_{i=2}^{m_{n}}\left(1+\left|\xi_{i}^{(n)}\right|+\ldots+\left|\xi_{i}^{(n)}\right|^{r}\right) \\
\leqslant \max \left(2^{(r+1)\left(m_{n}-1\right)},\left\{2 \max \left(\left|\xi_{i}^{(n)}\right| r\right)\right\}^{m_{n}-1}\right),
\end{gathered}
$$

the last inequality being obtained by the argument used in proving (6). By the lemma this last quantity does not exceed $\left(2^{r+1} H^{r}\left(F_{n}\right)\right)^{m-1}$. Hence if $F_{n}\left(\vartheta_{i}^{(\nu)}\right) \neq 0$ for $i=1, \ldots, r$, then

$$
\left|f_{v}\left(\xi^{(n)}\right)\right| \geqslant \frac{c_{10}}{H^{r m}\left(F_{n}\right) H^{m-1}\left(f_{\nu}\right)} .
$$

JOUR. 110. 
Since $\left\{f_{v}\right\}$ is an $L$-sequence for $\xi$, we can write

$$
\left|f_{\nu}(\xi)\right|=H^{-\gamma_{\nu}}\left(f_{\nu}\right)
$$

where $\gamma_{\nu} \rightarrow \infty$ with $\nu$. But from (9),

or

$$
\begin{gathered}
\left|f_{\nu}(\xi)\right|=\left|f_{\nu}\left(\xi^{(n)}+t_{n} H^{-\omega_{n} / m}\left(F_{n}\right)\right)\right|, \\
\left|f_{\nu}(\xi)\right|=\left|f_{\nu}\left(\xi^{(n)}\right)\right|+t_{n, \nu} H\left(f_{\nu}\right) H^{-\omega_{n} / m}\left(F_{n}\right),
\end{gathered}
$$

where $\left|t_{n, \nu}\right|<c_{11}$ for all $n$ and $v$.

For each sufficiently large $v$, there is an $n$ such that

$$
H\left(F_{n}\right) \leqslant H\left(f_{\nu}\right)<H\left(F_{n-1}\right) ;
$$

hereafter let $n$ be so defined as a function of $\nu$. Let $\epsilon$ be positive; we consider two cases, according as the inequality

$$
H\left(f_{v}\right)<H^{\omega_{n}}\left\{\left\{m^{2}(r+1)+e\right\}\left(F_{n}\right)\right.
$$

is true or false. Suppose first that (14) holds for infinitely many $\nu$. Then for such $\nu$,

$$
\frac{c_{10}}{H^{r m}\left(F_{n}\right) H^{m-1}\left(f_{v}\right)}>\frac{c_{10}}{H^{r m+m-1}\left(f_{v}\right)}
$$

and

$$
\frac{c_{11} H\left(f_{v}\right)}{H^{\omega_{n} / m}\left(F_{n}\right)}<\frac{c_{11}}{H^{r m+m-1+(e / m)}\left(f_{v}\right)} .
$$

But (11), (12), (13), (15) and (16) are incompatible for large $v$; hence for large $\nu$ for which (14) holds, $F_{n}\left(\vartheta_{i}^{(\nu)}\right)=0$ for some $i$, which implies that $F_{n} \mid f_{\nu}$.

Now consider the case where (14) is false, so that

$$
H^{\omega_{n} /\left\langle m^{2}(r+1)+\epsilon\right\}}\left(F_{n}\right) \leqslant H\left(f_{v}\right)<H\left(F_{n+1}\right) .
$$

Then by hypothesis 5 ,

$$
\frac{1}{H^{\gamma_{\nu}}\left(f_{v}\right)} \leqslant \frac{1}{H^{\gamma_{v} \omega_{n} /\left\langle m^{2}(r+1)+e\right\}}\left(F_{n}\right)}<\frac{1}{H^{\delta \gamma_{\nu} /\left(m m^{2}(r+1)+\tau\right)}\left(F_{n+1}\right)}
$$

and

$$
\frac{c_{10}}{H^{r m}\left(F_{n+1}\right) H^{m-1}\left(f_{\nu}\right)}>\frac{c_{10}}{H^{r m+m-1}\left(F_{n+1}\right)} .
$$

Moreover,

$$
\frac{c_{11} H\left(f_{\nu}\right)}{H^{\omega_{n+1} / m}\left(F_{n+1}\right)}<\frac{c_{11}}{H^{\left(\omega_{n+1} / m\right)-1}\left(F_{n+1}\right)} \text {. }
$$

But (11)-(13), with $n$ replaced by $n+1$, and (18)-(20) are again incompatible for large $\nu$, so that if (17) holds for large $\nu$ then $F_{n+1}\left(\vartheta_{i}^{(\nu)}\right)=0$ for some $i$, and so $F_{n+1} \mid f_{v}$. The proof is complete.

Corollary. A sufficient condition that $\xi$ belong to $U_{l}$ is that there be a sequence $\left\{F_{n}\right\}$ as described in Theorem 4 for which $\lim \inf m_{n}=l$. 
This result can be used to demonstrate the following basic fact:

Theorem 5. $U_{m}$ is not empty for any $m \geqslant 1$.

Let $P_{k}$ be a sequence of primes, with $\log P_{k+1} / \log P_{k}$ increasing monotonically and without bound, so that

$$
\zeta=\sum_{k=1}^{\infty} 2^{-P_{k}}
$$

is a Liouville number. Put

$$
q_{n}=2^{P_{n}}, \quad p_{n}=q_{n} \sum_{k=1}^{n} 2^{-P_{k}} ;
$$

then $\left\{q_{n} x^{m}-p_{n}\right\}$ is an $L_{m^{-}}$-sequence for $\zeta^{1 / m}$, and for this sequence the hypotheses of Theorem 4 are easily verified. Hence $U_{m}$ contains $\zeta^{1 / m}$, and so is not empty.

3. We shall say that the Liouville number $\xi$ with convergents $P_{n} / Q_{n}$ is strong if $\left\{Q_{n} x-P_{n}\right\}$ is an $L_{1}$-sequence for $\xi$, and weak if every $L_{1}$-sequence for $\xi$ is a proper subsequence of $\left\{Q_{n} x-P_{n}\right\}$. Thus if $\left|Q_{n} \xi-P_{n}\right|=Q_{n}^{-\kappa_{n}}$, then $\xi$ is strong or weak according as lim inf $\kappa_{n}$ is infinite or finite (of course $\lim \sup \kappa_{n}=\infty$, since $\xi$ is Liouville). The number $\zeta$ considered in the proof of Theorem 5 is a. strong Liouville number, while the numbers

$$
\zeta^{\prime}=\sum_{n=1}^{\infty}\left(10^{-(2 n) !}+10^{-3(2 n) !}+\ldots+10^{-3^{n^{\prime}}(2 n) !}\right),
$$

and

$$
\zeta^{\prime \prime}=\sum_{n=1}^{\infty}\left(10^{-(2 n+1) !}+10^{-3(2 n+1) !}+\ldots+10^{-3^{n^{\prime \prime}}(2 n+1) !}\right),
$$

where $3^{n^{\prime}} \leqslant 2 n<3^{n^{\prime}+1}$ and $3^{n^{\prime \prime}} \leqslant 2 n+1<3^{n^{\prime \prime}+1}$, are weak, since there are infinitely many $n$ for which each of the inequalities

$$
\left|\zeta^{\prime}-\frac{P_{n}^{\prime}}{Q_{n}^{\prime}}\right|>\frac{1}{Q_{n}^{\prime 3}}, \quad\left|\zeta^{\prime \prime}-\frac{P_{n}^{\prime \prime}}{Q_{n}^{\prime \prime}}\right|>\frac{1}{Q_{n}^{\prime \prime 3}}
$$

holds. It is clear that if $\alpha_{0}, \ldots, \alpha_{m-1}$ are strong Liouville numbers, then the monotonic union sequence formed from the denominators of their convergents is a large-gap sequence; that this is not necessarily true if the $\alpha^{\prime}$ 's are not strong is seen by taking $m=2, \alpha_{0}=\zeta^{\prime}, \alpha_{1}=\zeta^{\prime \prime}$.

We say that $\alpha_{0}, \ldots, \alpha_{m-1}$ are comparable Liouville numbers if there is an increasing sequence $\left\{N_{n}{ }^{\prime}\right\}$ of positive integers such that

$$
\limsup _{n \rightarrow \infty} \frac{\log \max _{k}\left(Q_{k}^{\left(h_{k}\left(N_{n}{ }^{\prime}\right)\right)}\right)}{\log \min _{k}\left(Q_{k}^{\left(h_{k}\left(N_{n}^{\prime}\right)\right)}\right)}<\infty,
$$

where $h_{k}(N)$ is defined as in the proof of Theorem 2. If $\alpha_{0}, \ldots, \alpha_{m-1}$ are both strong and comparable, it is easily seen that the sequence $\left\{N_{n}\right\}$ of Theorem 2 and the new sequence $\left\{N_{n}{ }^{\prime}\right\}$ may be taken to be identical. Suppose that $\left\{M_{n}\right\}$ is both an $N$ - and an $N^{\prime}$-sequence, and that $\left\{f_{n}^{* *}\right\}$ is the 
corresponding polynomial sequence, and suppose that no sequence $\left\{\bar{M}_{n}\right\}$ is both an $N$ - and an $N^{\prime}$-sequence, where $\left\{\bar{M}_{n}\right\}$ is the monotonic sequence formed by adjoining to $\left\{M_{n}\right\}$ an infinite number of elements to which correspond polynomials $\bar{f}^{*}$ distinct from all the $f^{*}$. Then clearly

$$
h_{k}\left(M_{n+1}\right)=h_{k}\left(M_{n}\right)+1
$$

for $n$ large and $0 \leqslant k \leqslant m-1$, and it is straightforward to show that both

$$
\frac{\log H\left(f_{n}^{*}\right)}{\log \min _{k}\left(Q_{k}^{\left(h_{k}\left(M_{n}\right)\right)}\right)} \text { and } \frac{\log H^{\omega_{n}}\left(f_{n}^{*}\right)}{\log \min _{k}\left(Q_{k}^{\left(h_{k}\left(M_{n}\right)+1\right)}\right)}
$$

are bounded away from zero and infinity, and so, taking $F_{n}=f_{n}^{*}$, it is easily verified that all the hypotheses of Theorem 4, except possibly the third, are satisfied. The following result then follows from Theorems 3 and 4 :

THeORem 6. Suppose that $\alpha_{0}, \ldots, \alpha_{m-1}$ are comparable strong Liouville numbers or rational numbers, one at least being irrational, and suppose that $\alpha_{0}+\ldots+\alpha_{m-1} \xi^{m-1}+\xi^{m}=0$. Then $\xi \varepsilon U_{m}$ if and only if every $L_{m}$-sequence $\left\{f_{n}^{*}\right\}$ for $\xi$ has only finitely many reducible elements.

The relation of comparability [defined by (21) for arbitrary increasing sequences $\left\{Q_{k}^{(n)}\right\}$ ] is rather difficult to work with, since it is not transitive. For example $\left\{10^{n}\right\}$ and $\left\{10^{(2 n) !}\right\}$ are comparable, as are $\left\{10^{n !}\right\}$ and $\left\{10^{(2 n+1) !}\right\}$, but $\left\{10^{(2 n) !}\right\}$ and $\left\{10^{(2 n+1) !}\right\}$ are not. The concept was introduced by Maillet [3] who showed that if $\alpha, \beta$ are comparable Liouville numbers then each of $\alpha \pm \beta, \alpha \beta$ and $\alpha / \beta$ is either a Liouville number or rational, and those which are Liouville are comparable to each other and to $\alpha$ and $\beta$. (Unfortunately, he neglected the non-transitivity of comparability, and concluded that the set of all Liouville numbers comparable with a given one, together with the rationals, forms a field.)

That some restriction is necessary in Theorem 6 , in addition to the requirement that $\alpha_{0}, \ldots, \alpha_{m-1}$ be Liouville numbers, is shown by a theorem recently proved by $P$. Erdös, to the effect that every real number is the root of an algebraic equation with Liouville or rational coefficients.

4. The following transcendence criterion is due to T. Schneider in the case where $q_{n}^{\prime \prime}=1$; Mahler pointed out that the proof applies in the more general theorem ( $c f$. [4] for a detailed proof):

Suppose that $\vartheta$ is a real number, and that there is a constant $\kappa$ and an infinite sequence of fractions $p_{n} / q_{n}$ whose denominators increase monotonically, such that

$$
\left|\vartheta-\frac{p_{n}}{q_{n}}\right|=q_{n}^{-\kappa_{n}} \quad \text { and } \quad \limsup _{n \rightarrow \infty} \frac{\log q_{n+1}}{\log q_{n}}<\infty .
$$


Then $\vartheta$ is transcendental if either of the following conditions is satisfied:

(i) $\kappa_{n} \geqslant \kappa>2$;

(ii) $\kappa_{n} \geqslant \kappa>1$ and, for each $n, q_{n}$ can be represented as a product $q_{n}{ }^{\prime} q_{n}^{\prime \prime}$, where $q_{n}{ }^{\prime}$ is a power of the fixed integer $q$, and

$$
\lim _{n \rightarrow \infty} \frac{\log q_{n}^{\prime \prime}}{\log q_{n}}=0 .
$$

On account of this theorem, it is of some interest to consider the numbers $\vartheta$ having approximations satisfying (22). If in Theorem $4, F_{n}(x)$ is taken to be $q_{n} x-p_{n}$, and $\xi$ is replaced by $\vartheta$ and $\omega_{n}$ by $\kappa_{n}$, then all hypotheses except (8) are fulfilled if $\kappa_{n}$ remains bounded as $n \rightarrow \infty$; that this is the case is an easy consequence of (22). But an examination of the proof shows that the conclusion still holds if

$$
\liminf _{n \rightarrow \infty} \kappa_{n}>m^{2}(r+1) \text {. }
$$

Since here the polynomials $F_{n}$ are linear, $m=1$ and we have the following theorem:

THEOREM 7. If $\vartheta$ is a real number having a sequence of approximations $p_{n} / q_{n}$ satisfying (22), where for all $n$

$$
r+1+\epsilon<\kappa_{n} \quad(r \text { an integer, } \epsilon>0),
$$

then $\vartheta \& U_{1} \cup \ldots \cup U_{r}$.

\section{Bibliography.}

1. K. Mahler, "Zur Approximation der Exponentialfunktion und des Logarithmus, I" $J$. reine angew. Math., 166 (1932), 118-136.

2. O. Perron, Die Lehre von den Kettenbrüchen (Leipzig, 1929).

3. E. Maillet, Theorie des nombres transcendants (Paris, 1906).

4. K. Mahler, "Ein Analogon zu einem Schneiderschen Satz", Proc. Royal Acad. Amsterdam, 39 (1936), 633-640 and 729-739.

University of Michigan, Ann Arbor,

Michigan, U.S.A.

\section{ON THE LATTICE DETERMINANTS OF TWO PARTICULAR POINT SETS}

\section{K. MAHLER*.}

It is well known that the star domain

$$
K:|x y| \leqslant 1
$$

\footnotetext{
* Received 14 May, 1952; read 15 May, 1952.
} 